\title{
Remain Aggressive Behavior in Children with Temporal Lobe Epilepsy after Surgery
}

\author{
Sayuri Sukigara', Eiji Nakagawa1, Masanori Ishikawa², Kenji Sugai', Masayuki Sasaki", \\ Takanobu Kaido ${ }^{3}$, Akio Takahashi ${ }^{3}$, Taisuke Otsuki ${ }^{3}$ \\ ${ }^{1}$ Department of Child Neurology, National Center Hospital, National Center of Neurology and Psychiatry \\ (NCNP), Tokyo, Japan \\ ${ }^{2}$ Department of Psychiatry, NCNP, Tokyo, Japan \\ ${ }^{3}$ Department of Neurosurgery, NCNP, Tokyo, Japan \\ Email: nakagawa@ncnp.go.jp
}

Received 29 December 2014; accepted 28 February 2015; published 2 March 2015

Academic Editor: Ana Adan, University of Barcelona, Spain

Copyright (C) 2015 by authors and Scientific Research Publishing Inc.

This work is licensed under the Creative Commons Attribution International License (CC BY).

http://creativecommons.org/licenses/by/4.0/

(c) (i) Open Access

\begin{abstract}
Behavioral problems have a high rate in patients with temporal lobe epilepsy but there have been few detailed reports about pediatric patients. We report two children with temporal lobe epilepsy who manifest behavior abnormality remaining after surgery. One child developed complex partial seizures at 10 years and 3 months of age caused by a left temporal ganglioglioma and manifested behavioral problems of lability, aggression, impulsivity and disinhibition. Seizures were relieved after temporal lobe resection at 10 years and 8 months of age but behavioral symptoms had been remained. The second child developed epilepsy at 3 years of age. He manifested similar behavioral problems at 7 years old. Seizures were relieved after temporal lobe resection at 13 years old but behavioral symptoms had been remained. Their courses suggested that aggressive behavior might be associated with not only temporal lesion but also other lesions such as frontal lobe or neural circuitry between both lobes. It is important to inform families of patients that behavioral disorders may not improve after epileptic surgery.
\end{abstract}

\section{Keywords}

Temporal Lobe Epilepsy, Behavioral Disorder, Aggression, Epileptic Surgery

\section{Introduction}

Aggressive and antisocial behavior of children in society and home is the source of concern. Those symptoms

How to cite this paper: Sukigara, S., Nakagawa, E., Ishikawa, M., Sugai, K., Sasaki, M., Kaido, T., Takahashi, A. and Otsuki, T. (2015) Remain Aggressive Behavior in Children with Temporal Lobe Epilepsy after Surgery. Journal of Behavioral and Brain Science, 5, 81-87. http://dx.doi.org/10.4236/jbbs.2015.53007 
are considered to be associated with later antisocial behaviors, adult criminality [1]. Meanwhile, behavioral abnormalities are often seen in patients with temporal lobe epilepsy [2]-[4]. Families of patients who undergo epileptic surgery are likely to expect to get relief from their children's behavioral problems with seizure recovery, especially when symptoms consist of antisocial behaviors such as aggression and disinhibition. Though there are many reports about adult epileptic patients with temporal lobe lesion accompanied by aggressive behavior undergoing surgery, there is a dearth of researches about children. Furthermore, little has become identified about pathogenesis of occurring behavioral abnormalities associated with structural brain abnormalities. Much more reports especially about children are needed to inform families of reliable prognostic prediction for not only seizure recovery but also recovery from behavioral problems. Here we report 2 children with temporal lobe epilepsy who manifest behavioral problems of lability, aggression, disinhibition and impulsivity. They underwent resection of structural lesions and both cases had behavioral symptoms remained even after seizures were relieved. We describe their courses and also presume pathogenesis of their behaviors from their findings and previous researches.

\section{Cases Reports}

\subsection{Case 1}

Patient 1 was the first child of unrelated parents. His mother had panic disorder and medically treated. Pregnancy, delivery, and neonatal history were normal. From his infancy, he was noticed to have awkwardness, difficulty in communicating with his friends, and tendency to play by himself. He had impulsivity and was easy to get angry or cry. He was diagnosed as autism spectrum disorders at 10 years old. Epilepsy had onset at 10 years and 3 months of age. Seizures consisted of loss of consciousness, rolling of the eyes, and unfocused status without response, lasting for several tens of seconds. Seizures recurred as often as several times per day. He started to take medicine. Routine blood examination was normal. Electroencephalogram (EEG) revealed abnormal spikes and sharp waves over the bilateral frontal areas and he started to take antiepileptic drugs. His lability and impulsivity got worsened around the same time, lashed out to the street, tried to jump from the balcony, raged, punched and kicked his mother and little sisters. Those behaviors were likely to be triggered by some complaint or anger. He also presented encopresis and enuresis. Brain magnetic resonance imaging (MRI) showed high intensity lesion on the left temporal lobe which involved hippocampus and amygdala (Figure 1(a)). Interictal ethyl cysteine dimer (ECD)-single-photon emission computed tomography (SPECT) showed no laterality of cerebral blood flow (CBF) on temporal lobe and CBF was obtained on whole cortex (Figure 2(a)). EEG revealed occasional spikes and sharp waves over the bilateral frontal areas during interval stage and no remarkable spikes were found during attacks (Figure 3(a)). Left temporal resection was performed at 10 years and 8 months of age (Figure 1(a)). Pathological examination of resected material confirmed the diagnosis of ganglioglioma. Seizure remained postoperatively at a rate of one a day and started to take sodium valproate. Behavioral abnormal-
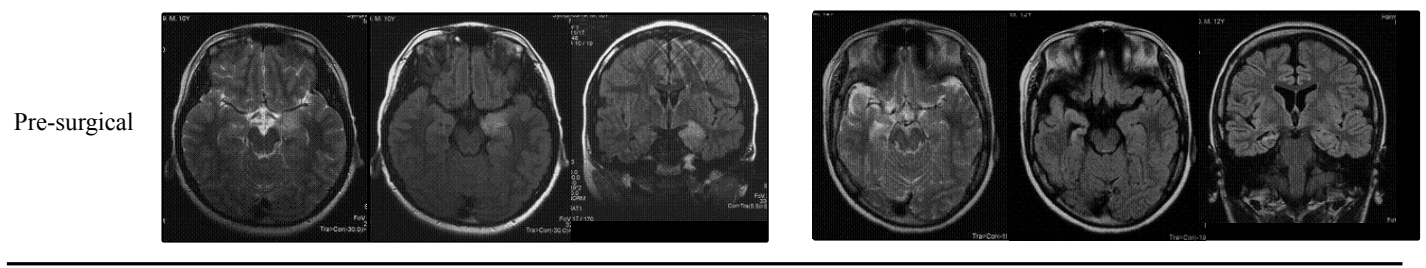

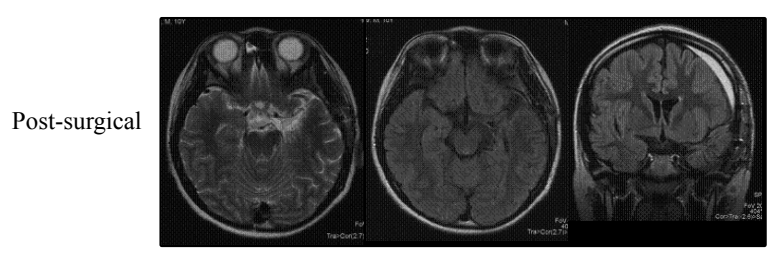

(a)

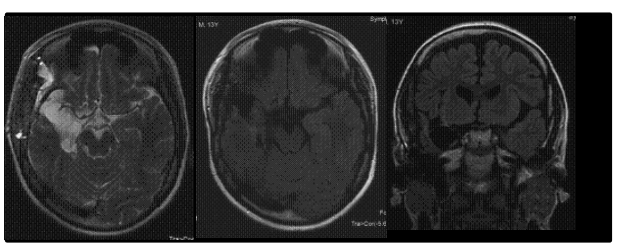

(b)

Figure 1. Brain MRI. (a) Patient 1, Brain MRI showing a high intensity lesion in the left medial temporal lobe on T2-weighted (left) and FLAIR (right) images. The lesion was resected; (b) Patient 2, Brain MRI showing a high intensity lesion in the right medial temporal lobe on T2-weighted (left) and FLAIR (right) images. The lesion was resected. 


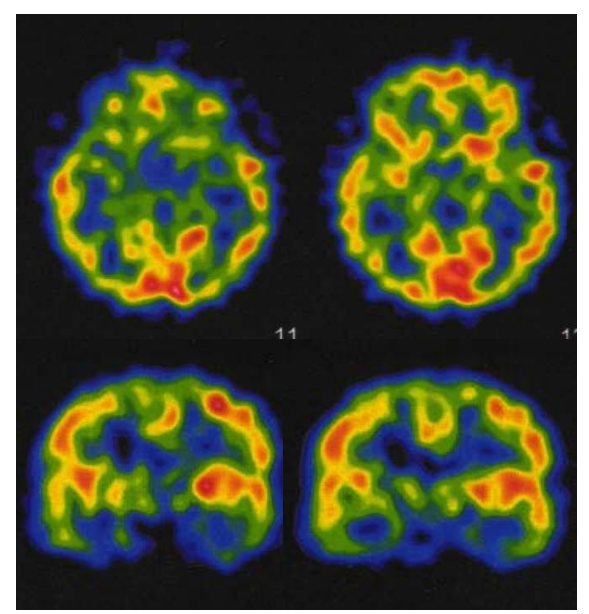

(a)

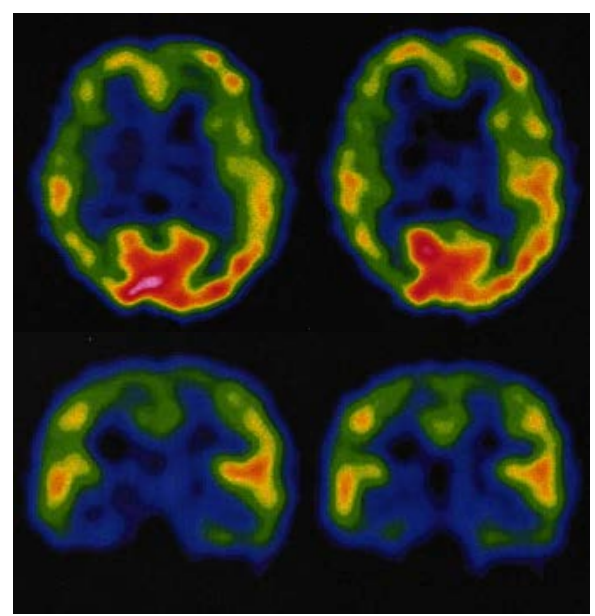

(b)

Figure 2. Interictal SPECT. (a) Patient 1, Interictal ECD-SPECT showing no laterality of CBF on temporal lobe and left-dominant increase on whole cortex and basal ganglia; (b) Patient 2, Interictal EMZ-SPECT showing decrease of $\mathrm{CBF}$ on the right temporal lobe.
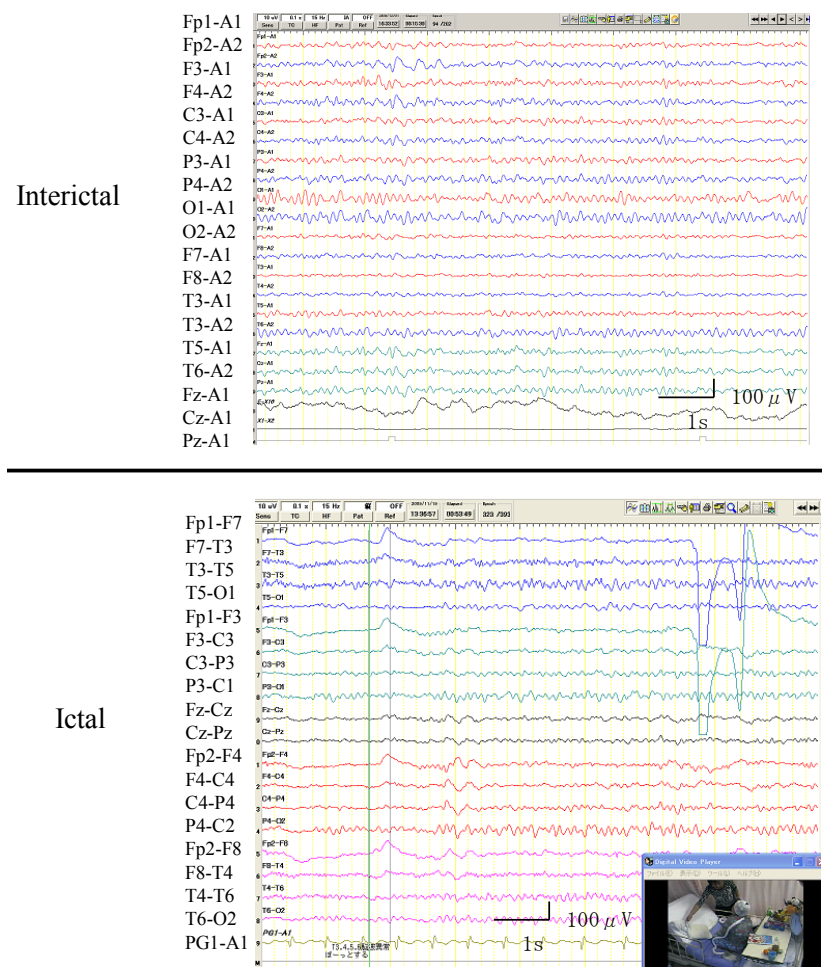

(a)

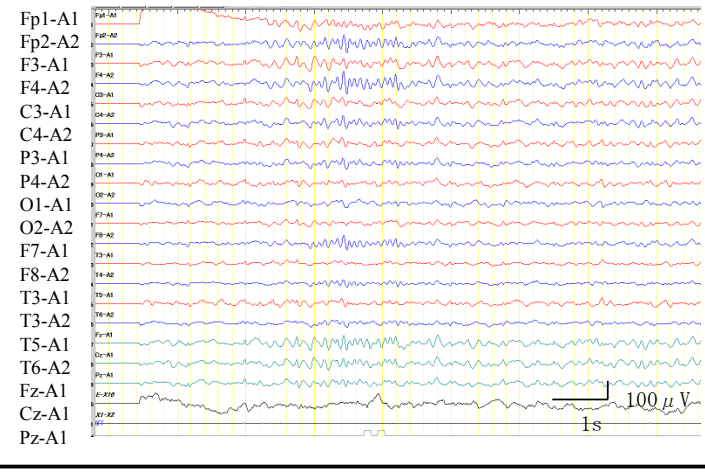

Fp1-A1 F32-A2 1 F4-A2 C3-A C4-A2 P3-A1 $\mathrm{P} 4-\mathrm{A} 2$ O1-A1 $\mathrm{O} 2-\mathrm{A} 2$ F7-A1 F8-A2 T3-A1 T3-A2 T5-A1 T6-A2 Fz-A1 Cz-A1 Pz-A1 1

Figure 3. EEG. (a) Patient 1, Interictal EEG showing occasional spikes and sharp waves over the bilateral frontal area and ictal EEG showing no remarkable spikes; (b) Patient 2, Interictal EEG showing occasional small spikes over the right frontocentral areas and ictal EEG showing frequent rhythmic waves over the right frontal areas.

ity such as lability, aggression, impulsivity and disinhibitation got worsened after the surgery. He blasted a name of a character of cartoon. He often needed to be suppressed with intramuscular injection of diazepam or risperidone orally and was placed in psychiatric ward for 20 days. At last, he was transferred to specialized hospital of child psychiatry. The result of pre- and post-operative Wechsler Intelligence Scale for Children-Third Edition (WISC-III) is as followed (Figure 4(a)). 


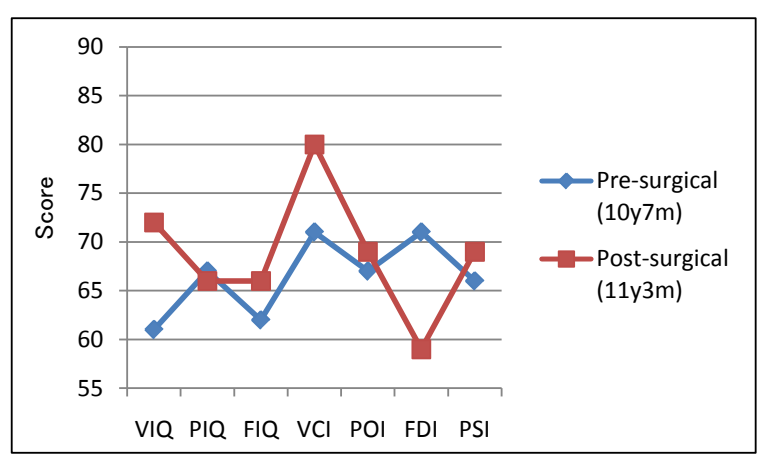

(a)

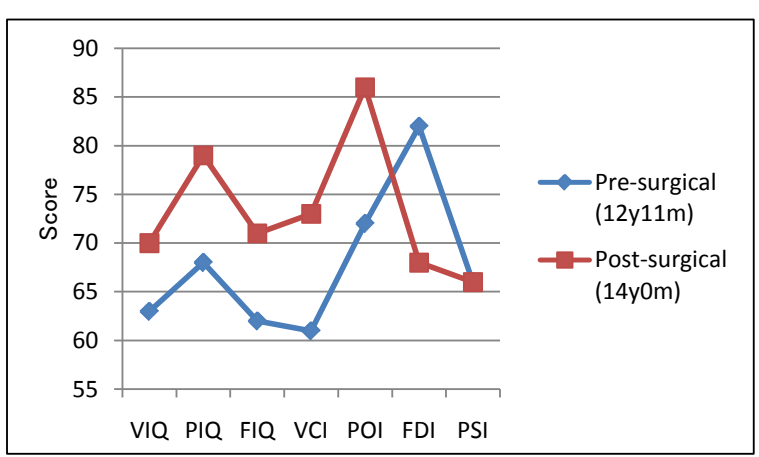

(b)

Figure 4. WISC-III. (a) Patient 1; (b) Patient 2. Abbreviation: VIQ, Verbal Intelligence Quotient; PIQ, Performance Intelligence Quotient; FIQ, Full Scale Intelligence Quotient; VCI, Verbal Comprehension Index; POI, Perceptual Organization Index; FDI, Freedom from Distractibility Index; PSI, Processing Speed Index. In both patients, post-operative scores of WISC-III showing improvement of VIQ, VCI and FIQ.

\subsection{Case 2}

Patient 2 was the first child of unrelated healthy parents. He was born at 38 weeks gestation. He needed a few minutes to start to cry due to rotation abnormality and loop of the umbilical cord, but following course was good, discharging normally. From his infancy, he liked to line up toy-cars. Epilepsy had onset at 3 years and 5 months of age. Seizures consisted of left sided clonic seizure and deviation of eyes to left lasting for several minutes. Seizures recurred as often as several times per day. Though no abnormal spikes were seen on the EEG, he started to take medicine. He also presented steroid-resistant nephrotic syndrome at 3 years and 10 months, diagnosed as focal glomerulonephritis, and was treated with steroid pulse therapy. He developed cardio pulmonary arrest due to hypertension encephalopathy on third day of steroid therapy and was resuscitated followed by mechanical ventilation for 2 days. Seizure for several times a month remained after he recovered, which consisted of left deviation of both eyes, left turning of the head, cyanosis and vomiting. He continued to take sodium valproate but seizure was not controlled. He had difficulty in communicating with others in the kindergarten. After entering a regular class in the elementary school, he could not follow classes and refused to go to school. He was also concerned of behavioral problems such as hyperactivity, rage attacks, disinhibition, lability, and impulsivity especially after starting to take clobazam. He also had verbal developmental delay and diagnosed as developmental disorder which could not identified as attention-deficit hyperactivity (ADHD), Asperger syndrome, or learning disorders (LD). Behavioral problems improved after quitting taking clobazam. At the 9 years of age (4th year of school), he started to go to special class. At 12 years old, seizures increased as often as 1 - 2 times per day. He was admitted to our hospital for examinations. Brain MRI showed high intensity lesion on the right temporal lobe (Figure 1(b)). Magnetoencephalography (MEG) revealed clusters of dipoles on the right frontal perculum and interictal Iomazenil-SPECT showed decrease of CBF on the right temporal lobe (Figure 2(b)). Small spikes were occasionally seen over the right fronto-central areas on the interictal EEG, and rhythmic waves were often seen over the right frontal areas were observed during attacks (Figure 3(b)). He was diagnosed as right temporal lobe epilepsy. Right temporal lobectomy was performed at 13 years old (Figure 1(b)). Pathological examination of resected material confirmed the diagnosis of hippocampal sclerosis and focal cortical dysplasia (FCD) type IA. Seizure resolved after the surgery, but behavioral abnormality such as aggression, impulsivity and disinhibition remained and often made him hit his little sisters. After discharge, he has been continued to be followed as our out-patient. The result of pre- and post-operative WISC-III is as followed (Figure 4(b)).

\section{Discussion}

These two children described behavioral problems accompanied by temporal lobe epilepsy, which remained after temporal lobe resection though seizure was resolved. They showed similar findings in their courses and examinations (Table 1). Behavioral problems seen in both patients consisted of lability, aggression, and impulsivity. They also had same MRI lesions which were medial temporal areas including amygdala and hippocampus, 
Table 1. Clinical features of our cases.

\begin{tabular}{|c|c|c|}
\hline & Case 1 & Case 2 \\
\hline Sex & Male & Male \\
\hline Onset of seizure & 10 y $3 \mathrm{~m}$ & 3 y $5 \mathrm{~m}$ \\
\hline Onset of behavioral disorder & Infancy & Infancy \\
\hline Behavioral symptoms & $\begin{array}{l}\text { Lability, aggression, } \\
\text { Disinhibitation and impusivity }\end{array}$ & $\begin{array}{l}\text { Lability, aggression, } \\
\text { disinhibitation and impusivity }\end{array}$ \\
\hline Side of surgery & Left & Right \\
\hline Preoperative interictal EEG & $\begin{array}{l}\text { Occasional spikes and sharp waves } \\
\text { over the bilateral frontal area }\end{array}$ & $\begin{array}{l}\text { Occasional small spikes over the right } \\
\text { fronto-central areas }\end{array}$ \\
\hline Ictal EEG & No ictal spikes & Frequent rhythmic waves over the right frontal areas \\
\hline Pathological diagnosis & Ganglioglioma & Hippocampal sclerosis \& FCD type IA \\
\hline Seizure outcome ${ }^{*}$ & Class III & Class I \\
\hline Full scale IQ & Improved & Improved \\
\hline Verbal IQ & Improved & Improved \\
\hline Performance IQ & Improved & Improved \\
\hline
\end{tabular}

*Engel (1987) postoperative seizure outcome: class I, seizure freedom; class II, rare seizures; class III, significant improvement in seizure control; class IV, no change or deterioration in seizure control. FCD: focal cortical dysplasia.

and the lesions were consistent with those characteristic behaviors. Many studies with antisocial and aggressive adults have found brain abnormalities in temporal areas but there is a dearth of studies about correlation between aggressive behavior and brain abnormalities especially about children. One study indicated reduced right temporal lobe and right temporal grey matter volume within participants aged 9 - 20 years with conduct disorder (CD) [5]. The right temporal lobe is thought to be associated with empathy and structural abnormality in temporal lobe causes the deficiency of empathy associated with aggressive behavior [6].

It is striking that some lesions in frontal lobe are also thought to be associated with aggressive behaviors, which are anterior cingulated cortex (ACC) and orbital frontal cortex. ACC is a part of limbic system structures along with amygdala. ACC is involved in the regulation of emotional behaviors during conflicts and decision making [7]. Sterzer et al. found that fMRI in CD group of participants showed less activation in the left amygdala and silent in the ACC while passively viewing negative pictures when compared to age-matched controls, 9 15 years old [8]. The result indicates impairment in amygdala and ACC in processing emotional cues in the environment. Emotional control is decreased and an inclination for impulsive aggression among individuals is increased among individuals with CD. Orbital frontal cortex is also involved in reactive aggression. Orbital frontal cortex is described as a part of neural circuitry with amygdala, medial hypothalamus, and dorsal periaqueductal gray. The amygdala modulates these systems as a function of the presence of the threat or appetitive cues in the environment. Orbital frontal cortex modulates these systems as a function of social emotional cues and knowledge of the position of con-specifics in the current dominant hierarchy [9].

In our both cases, MRI abnormalities were found in the left medial temporal lobe, but abnormal spikes were observed over the frontal areas not over the temporal areas on interictal EEG. The findings sometimes created the confusion at decision making of selecting surgical treatment or not. These findings might be caused by neural circuitry of frontal and temporal lobes.

In both cases, behavioral disorders remained even after temporal lobe resection regardless of improvement of IQ scores on WISC-III. Prognosis for psychotic symptoms in adult patients who undergo epileptic surgery has been discussed but little is done in children. In the previous study, psychiatric disorders were found in 50/60 $(83 \%)$ children who underwent temporal lobe resections and there was no significant relationship between pathology, sex, seizure frequency, or postoperative seizure outcome and psychiatric disorder, other than pervasive developmental disorder. The same proportion of children had psychiatric diagnosis pre- and post-operatively $(72 \%$ both) [10]. Janice et al reported psychiatric consequences of 14 cases including children after temporal lobectomy for intractable seizures. Two previously healthy women developed psychosis, previous psychiatric problems 
of four patients worsened, and one with explosive rage attacks preoperatively had marked improvement [11]. Moreover, less few reports of behavioral recovery after epileptic surgery have been reported. Neville reported 2 children with partial epilepsy who manifested social and language regression and partial recovery after surgical treatment [12]. The fact that resection of MRI lesion does not improve behavioral disorders reveals that symptoms are related with not only the structural lesion but also other functional lesions. Frontal lobe such as orbital frontal cortex or anterior cingulated cortex could be the functional lesion because of the neural circuitry as previously described.

Thus, behavioral disorder especially aggression is related with frontal and temporal lobes, acting by themselves or modulating each other.

\section{Conclusion}

We reported two children with behavioral disorders accompanied by temporal lobe epilepsy which remained after epileptic resection. Multiple lesions and systems modulating each other in temporal and frontal lobes are thought to be involved with aggressive behaviors. Resection of structural lesion does not always resolve behavioral problems like our cases. It is important to inform the possibility of remaining problems after surgery to families preliminarily.

\section{Conflict of Interest}

The authors declare that they have no conflict of interest.

\section{Acknowledgements}

This study was supported in part by Intramural Research Grant (24-8, 25-3, 25-6; Clinical Research for Diagnostic and Therapeutic Innovations in Developmental Disorders) for Neurological and Psychiatric Disorders of NCNP.

\section{References}

[1] Cappadocia, M.C., Desrocher, M., Pepler, D. and Schroeder, J.H. (2009) Contextualizing the Neurobiology of Conduct Disorder in an Emotion Dysregulation Framework. Clinical Psychology Review, 29, 506-518. http://dx.doi.org/10.1016/j.cpr.2009.06.001

[2] Rutter, M., Graham, P. and Yule, W. (1970) A Neuropsychiatric Study in Childhood. Clinics in Developmental Medicine. No.35/36. Spastics International Medical Publications (Mac Keith Press), London.

[3] Hoare, P. (1984) The Development of Psychiatric Disorder among Schoolchildren with Epilepsy. Developmental Medicine \& Child Neurology, 26, 3-13. http://dx.doi.org/10.1111/j.1469-8749.1984.tb04399.x

[4] Davies, S., Heyman, I. and Goodman, R. (2003) A Population Survey of Mental Health Problems in Children with Epilepsy. Developmental Medicine \& Child Neurology, 45, 292-295. http://dx.doi.org/10.1111/j.1469-8749.2003.tb00398.x

[5] Kruesi, M.J., Schmidt, M.E., Donnelly, M., Hibbs, E.D. and Hamburger, S.D. (2004) Urinary Free Cortisol Output and Disruptive Behavior in Children. Journal of the American Academy of Child \& Adolescent Psychiatry, 28, 441-443. http://dx.doi.org/10.1097/00004583-198905000-00024

[6] Perry, R.J., Rosen, H.R., Kramer, J.H., Beer, J.S., Levenson, R.L. and Miller, B.L. (2001) Hemispheric Dominance for Emotions, Empathy and Social Behavior: Evidence from Right and Left Handers with Frontotemporal Dementia. Neurocase, 7, 145-160.

[7] Bush, G., Luu, P. and Posner, M.I. (2000) Cognitive and Emotional Influences in Anterior Cingulated Cortex. Trend in Cognitive Sciences, 6, 215-21. http://dx.doi.org/10.1016/S1364-6613(00)01483-2

[8] Sterzer, P., Stadler, C., Krebs, A., Kleinschmidt, A. and Poustka, F. (2005) Abnormal Neural Responses to Emotional Visual Stimuli in Adolescents with Conduct Disorder. Biological Psychiatry, 57, 7-15. http://dx.doi.org/10.1016/j.biopsych.2004.10.008

[9] Blair, R.J.R. (2004) The Roles of Orbital Frontal Cortex in the Modulation of Antisocial Behavior. Brain \& Cognition, 55, 198-208. http://dx.doi.org/10.1016/S0278-2626(03)00276-8

[10] McLellan, A., Davies, S., Heyman, I., Harding, B., Harkness, W., Taylor, D., Neville, B.G.R. and Cross, J.H. (2005) Psychopathology in Children with Epilepsy before and after Temporal Lobe Resection. Developmental Medicine \& Child Neurology, 47, 666-672. http://dx.doi.org/10.1017/S0012162205001362 
[11] Janice, R.S. (1999) Psychiatric Consequences of Temporal Lobectomy for Intractable Seizures: A 20- - 30-Year Follow-Up of 14 Cases. Psychological Medicine, 20, 529-545.

[12] Neville, B.G.R., Harkness, W.F.J., Cross, J.H., Cass, H.C., Burch, V.C., Lees, J.A. and Taylor, D.C. (1997) Surgical Treatment of Severe Autistic Regression in Childhood Epilepsy. Pediatric Neurology, 16, 137-140.

http://dx.doi.org/10.1016/S0887-8994(96)00297-4 